\title{
Graphene sustained nonlinear modes in dielectric waveguides
}

\author{
Aldo Auditore, ${ }^{1}$ Costantino De Angelis, ${ }^{1, *}$ Andrea Locatelli, ${ }^{1}$ Stefano Boscolo, ${ }^{2}$ Michele Midrio, ${ }^{2}$ \\ Marco Romagnoli, ${ }^{3}$ Antonio-Daniele Capobianco, ${ }^{4}$ and Gianfranco Nalesso ${ }^{4}$ \\ ${ }^{1}$ Dipartimento di Ingegneria dell'Informazione, Università degli Studi di Brescia, Brescia 25123, Italy \\ ${ }^{2}$ Dipartimento di Ingegneria Elettrica, Gestionale e Meccanica, Università degli Studi di Udine, Udine 33100, Italy \\ ${ }^{3}$ CNIT National Laboratory of Photonic Networks, Pisa 56124, Italy \\ ${ }^{4}$ Dipartimento di Ingegneria dell'Informazione, Università degli Studi di Padova, Padova 35131, Italy \\ *Corresponding author: costantino.deangelis@ing.unibs.it
}

Received December 17, 2012; accepted January 16, 2013;

posted January 23, 2013 (Doc. ID 181824); published February 21, 2013

\begin{abstract}
We discuss the existence of nonlinear modes sustained by graphene layers in dielectric waveguides. Taking advantage of the almost two dimensional nature of graphene, we introduce the nonlinear effect as a parameter in the continuity equations. We then apply our modeling to a simple slab waveguide to enlighten how graphene can be used to induce huge nonlinear phase shifts at easily accessible power levels. (c) 2013 Optical Society of America OCIS codes: $190.4390,130.0130$.
\end{abstract}

After its experimental isolation from bulk graphite [1], graphene has attracted increasing interest from the scientific community. Indeed, this single layer of carbon atoms packed into a honeycomb lattice reveals amazing and very useful properties that have already inspired a huge variety of devices embracing different areas ranging from electronics to optics [2-4]. As a matter of fact, the linear optical properties of graphene show interesting tunable features from the mid-infrared to the visible spectrum and a plethora of applications ranging from photovoltaics and light emitting devices to the realization of novel components have already appeared in the literature [3-9].

More recently the nonlinear optical properties of graphene have been enlightened both theoretically and experimentally [10-15]. Indeed graphene might have a very favorable figure of merit for nonlinear optical applications, with high nonlinear Kerr response together with an overall low absorption coefficient; thus this new material could take the scene in nonlinear photonics and pave the way toward potentially revolutionary applications. From the theoretical viewpoint, the modeling of graphene-based devices is not conveniently done by borrowing the results that have been previously obtained in the field of nonlinear guided waves using standard bulk nonlinear media [16]; graphene-based integrated optics asks for suited modeling techniques able to take full advantage of the almost two-dimensional nature of the very thin graphene layers. In this Letter, we will first define the theoretical approach in the analysis of nonlinear guided waves in graphene-based optical waveguides and then apply this technique to a simple slab waveguide used here as a prototype to determine (using experimentally accessible parameters) the typical values of the coefficients describing the intensity dependent nature of the modal wave number.

In Fig. 1 we report the basic geometry we will consider in this Letter: a single slab of silicon enriched nitride $\left(\epsilon_{r 1}=4.76\right)$ of width $2 s=400 \mathrm{~nm}$ is the waveguide core; the cladding is made of silica $\left(\epsilon_{r 2}=2.25\right)$ and at the boundaries between core and cladding we have a single graphene layer of thickness $d_{g}=0.34 \mathrm{~nm}$.
As far as the electromagnetic constants are concerned, we write the linear contribution to the relative complex permittivity of graphene as []ㅡ:

$$
\epsilon_{r C}=1+\frac{\sigma_{\Sigma, I}^{(1)}}{d_{g} \omega \epsilon_{0}}-i \frac{\sigma_{\Sigma, R}^{(1)}}{d_{g} \omega \epsilon_{0}}=\epsilon_{r C, R}+i \epsilon_{r C, I},
$$

where the surface complex conductivity $\sigma_{\Sigma}^{(1)}=\sigma_{\Sigma, R}^{(1)}+$ $i \sigma_{\Sigma, I}^{(1)}$ (in Siemens) is obtained from theoretical models now well established and experimentally validated $[\underline{17}, \underline{18}]$. To take into account the complex nonlinear contribution to the refractive index change we write:

$$
\Delta \epsilon_{r \operatorname{Kerr}}\left(\left|\vec{E}_{\|}\right|^{2}\right)=\chi^{(3)} \frac{\left|\vec{E}_{\|}\right|^{2}}{1+\frac{\left|\vec{E}_{\|}\right|^{2}}{\left|E_{\text {sat }}\right|^{2}}},
$$

where $\vec{E}_{\|}$is the electric field on the plane of the graphene layer and the values of the nonlinear coefficients $\left(\chi^{(3)}\right.$ and $\left.E_{\text {sat }}\right)$ are taken from the experimental data reported

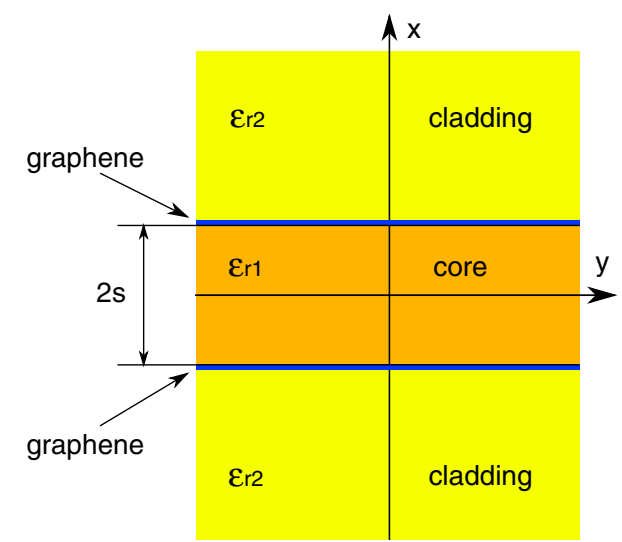

Fig. 1. (Color online) Schematic of the slab waveguide of total width 2s: the core (silicon enriched nitride) has permittivity $\epsilon_{r 1}=4.76$ and the cladding (silica) has permittivity $\epsilon_{r 2}=$ 2.25. At the boundaries between core and cladding we have the graphene layers of thickness $d_{g}=0.34 \mathrm{~nm}$. 
in [15]. In the nonlinear problem we will experience the sum of the linear and nonlinear contributions to give rise to a total surface complex relative permittivity written as:

$$
\epsilon_{r S}\left(\left|\vec{E}_{\|}\right|^{2}\right)=d_{g}\left(\epsilon_{r C}+\Delta \epsilon_{r \operatorname{Kerr}}\left(\left|\vec{E}_{\|}\right|^{2}\right)\right)
$$

At the graphene boundary we set the following conditions on the tangential components of the electromagnetic field:

$$
\begin{aligned}
\left(\vec{E}_{2}-\vec{E}_{1}\right) \times \hat{x} & =0 \\
\left(\vec{H}_{2}-\vec{H}_{1}\right) \times \hat{x} & =i \omega \epsilon_{0} \epsilon_{r S}\left(\left|\vec{E}_{\|}(x= \pm s)\right|^{2}\right) \vec{E}_{\|}(x= \pm s),
\end{aligned}
$$

where, thanks to the extremely small thickness of the graphene layer, the nonlinearity has become a parameter embedded into the coefficients describing the continuity of the tangential components of the electromagnetic field. Using the above described approach, the results we will present in the rest of the Letter have been obtained by modeling the graphene layer as a zero thickness nonlinear medium; however, we have always verified their soundness by resorting to COMSOL finite element simulations [19] where the graphene layer has a nonzero thickness of $0.34 \mathrm{~nm}$.

To describe mode propagation along $z$, we first note that, at first-order, the $y$ dependence of the electromagnetic field can be neglected; we then look for guided modes with harmonic temporal dependence $\exp (i \omega t)$ and spatial variation $\vec{E}_{1,2}(x, z), \vec{H}_{1,2}(x, z) \sim \exp (-i \beta z \pm$ $\left.\kappa_{1,2} x\right)$ with $\kappa_{1}^{2}=\beta^{2}-\epsilon_{r 1} k_{0}^{2}=-\kappa_{n}^{2}$ and $\kappa_{2}^{2}=\beta^{2}-\epsilon_{r 2} k_{0}^{2}=$ $\Gamma_{m}^{2}$. Obviously the complex wavenumber $\beta$, through its real and imaginary parts, describes the evolution of both the phase and the amplitude of the guided modes. We now apply the above modeling to derive the dispersion relation of TE and TM modes of the symmetric slab waveguide and we then solve them for $\lambda=1.55 \mu \mathrm{m}$; note that, since we have $\Re e\left(\epsilon_{r C}\right)>0$, graphene plasmons are forbidden here $[20,21]$. In the following we use the system parameters described in Fig. 1 and the graphene electromagnetic constants as obtained from Eqs. (1) and (2); in particular to determine the nonlinear constants we make reference to [15] to get a self-focusing Kerr coefficient $n_{2}=10^{-11} \mathrm{~m}^{2} / \mathrm{W}$, a two photon absorption coefficient $k_{2}=-5 \cdot 10^{-11} \mathrm{~m}^{2} / \mathrm{W}$ and a saturation of the nonlinear response $I_{\text {sat }}=6 \cdot 10^{10} \mathrm{~W} / \mathrm{m}^{2}$ [22] .

When dealing with TE modes, the only nonzero components of the electromagnetic field are $E_{y}, H_{x}$, and $H_{z}$; matching the field components at the corecladding interface and taking into account the graphene contributions we get: $H_{z 1}(x= \pm s)-H_{z 2}(x= \pm s)= \pm$ $i \omega \epsilon_{0} \epsilon_{r S}\left(\left|E_{y}(x= \pm s)\right|^{2}\right) E_{y}(x= \pm s)$. The dispersion relations of even and odd TE modes are then easily obtained and they read, respectively:

$$
\tan \left(\kappa_{n} s\right)=\frac{\Gamma_{m}}{\kappa_{n}}\left(1-\frac{\omega^{2} \mu_{0} \epsilon_{0} \epsilon_{r S}\left(\left|E_{y}(x= \pm s)\right|^{2}\right)}{\Gamma_{m}}\right),
$$

$$
\frac{1}{\tan \left(\kappa_{n} s\right)}=-\frac{\Gamma_{m}}{\kappa_{n}}\left(1-\frac{\omega^{2} \mu_{0} \epsilon_{0} \epsilon_{r S}\left(\left|E_{y}(x= \pm s)\right|^{2}\right)}{\Gamma_{m}}\right) .
$$

In Fig. 2 we report the solution of the dispersion relation of the fundamental TE mode, as a function of the peak intensity $I=-(1 / 2) E_{y}(x=0) H_{x}^{*}(x=0)$; since the graphene layer has $n_{2}>0$, the real part of the modal effective refractive index $\left(n_{e}=\Re e\left(\beta / k_{0}\right)\right)$ increases for increasing intensities. Remarkably peak intensities well below $0.1 \mathrm{GW} / \mathrm{cm}^{2}$ are sufficient to cause important changes: for $I=7 \mathrm{MW} / \mathrm{cm}^{2}$ we get $\Delta n_{e} \simeq 7.75 \cdot 10^{-4}$ and thus a phase change of $\pi$ is achieved for a device length of $L=1 \mathrm{~mm}$. In Fig. 2 the dashed curve corresponds to the imaginary part of the modal effective refractive index $\left(k=\mathfrak{s} m\left(\beta / k_{0}\right)\right)$; note that, having $k_{2}<0$, the graphene layer acts as a saturable absorber and the overall mode losses decrease for increasing intensities.

When dealing with TM modes, the only nonzero components of the electromagnetic field are $E_{x}, H_{y}$, and $E_{z}$; matching the field components at the core-cladding interface and taking into account the graphene contributions we get: $H_{y 1}(x= \pm s)-H_{y 2}(x= \pm s)=\mp i \omega \epsilon_{0} \epsilon_{r S}\left(\mid E_{z}(x=\right.$ $\left.\pm s)\left.\right|^{2}\right) E_{z}(x= \pm s)$. The dispersion relations of even and odd TM modes are then easily obtained and they read, respectively:

$$
\begin{aligned}
& \tan \left(\kappa_{n} s\right)=\frac{\epsilon_{r 1}}{\epsilon_{r 2}} \frac{\Gamma_{m}}{\kappa_{n}}\left(\frac{1}{1+\frac{\epsilon_{r S}\left(\left|E_{z}(x= \pm s)\right|^{2}\right) \Gamma_{m}}{\epsilon_{r 2}}}\right), \\
& \frac{1}{\tan \left(\kappa_{n} s\right)}=-\frac{\epsilon_{r 1}}{\epsilon_{r 2}} \frac{\Gamma_{m}}{\kappa_{n}}\left(\frac{1}{1+\frac{\epsilon_{r S}\left(\left|E_{z}(x= \pm s)\right|^{2}\right) \Gamma_{m}}{\epsilon_{r 2}}}\right) .
\end{aligned}
$$

In Fig. 3, we report the solution of the dispersion relation of the fundamental TM mode as a function of the peak intensity $I=(1 / 2) E_{x}(x=0) H_{y}^{*}(x=0)$; we find here similar features with respect to what we saw in Fig. 2: in Fig. 3 we note that peak intensities well below

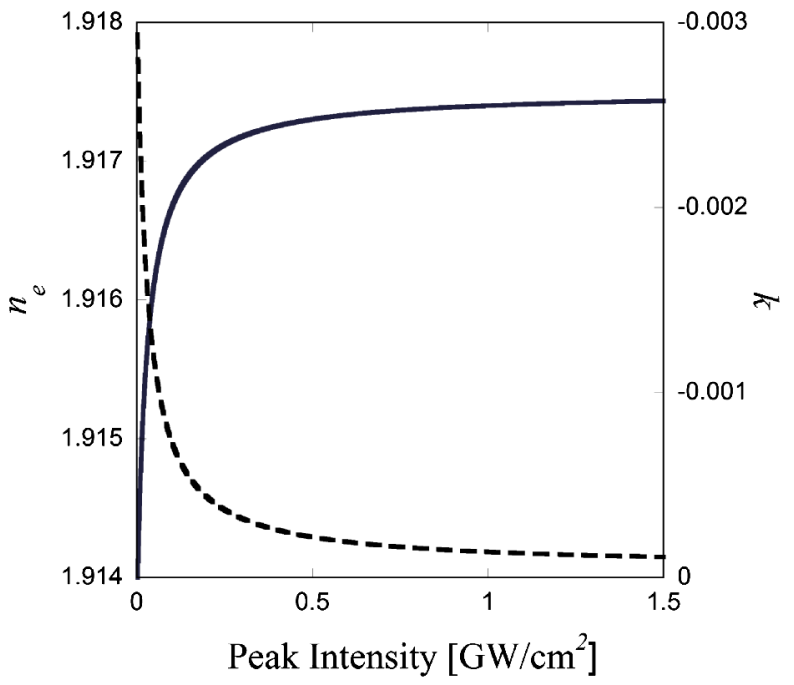

Fig. 2. Effective refractive index of the $\mathrm{TE}_{0}$ mode versus peak intensity I: continuous and dashed curves correspond to the real and imaginary part, respectively. 


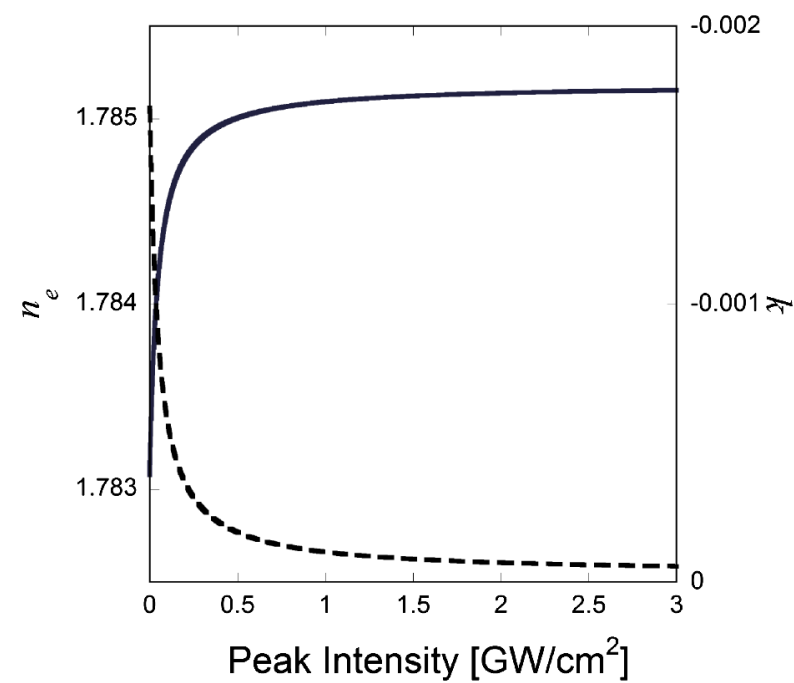

Fig. 3. Effective refractive index of the $\mathrm{TM}_{0}$ mode versus peak intensity I: continuous and dashed curves correspond to the real and imaginary part, respectively.

$0.1 \mathrm{GW} / \mathrm{cm}^{2}$ are sufficient to cause important changes on the real part of the modal effective refractive index $n_{e}$; however, it is interesting to observe that the TM case is less efficient than the $\mathrm{TE}$ case due to the fact that the nonlinear response depends on the longitudinal electric field $\left(E_{z}\right)$. In Fig. 3, the dashed curve shows the intensity dependent mode losses; the saturable absorption mechanism is clearly visible and the overall mode losses decrease for increasing intensities.

Obviously the approach we have presented here can be used also to analyze the behavior of different structures. As far as symmetrical one-dimensional waveguiding geometries are concerned, for example, a single graphene layer in the waveguide core center could prove useful in order to couple the nonlinear layer with the strongest electric field lines. Another very interesting situation corresponds to the one-dimensional asymmetric slab waveguide with a graphene layer located at the interface between the core and the upper cladding. Even more interesting situations could arise if one considers coupled waveguides where the possibility of tuning the wave number through the input intensities could be exploited for all optical beam shaping [23].

In conclusion, we have discussed nonlinear modes sustained by graphene layers in dielectric waveguides. We have first introduced the modeling technique where we have shown that graphene nonlinearity can be dealt with by mapping the nonlinear effect into a parameter in the boundary conditions; we have then applied our modeling to a simple slab waveguide and shown that graphene can be used to induce huge nonlinear phase shifts into guided modes. With experimentally accessible parameters we have proved the possibility to obtain $\pi$ phase shifts with very small footprint at easily accessible power levels.

AA, CDA, and AL acknowledge financial support from CARIPLO Foundation under grant no. 2010-0595 and U.S. ARMY under grant no. W911NF-12-1-0202.

\section{References}

1. K. S. Novoselov, A. K. Geim, S. V. Morozov, D. Jiang, Y. Zhang, S. V. Dubonos, I. V. Grigorieva, and A. A. Firsov, Science 306, 666 (2004).

2. F. Bonaccorso, Z. Sun, T. Hasan, and A. C. Ferrari, Nat. Photonics 4, 611 (2010).

3. A. Vakil and N. Engheta, Science 332, 1291 (2011).

4. M. Liu, X. Yin, and X. Zhang, Nano Lett. 12, 1482 (2012).

5. M. Midrio, S. Boscolo, M. Moresco, M. Romagnoli, C. De Angelis, A. Locatelli, and A.-D. Capobianco, Opt. Express 20, 23144 (2012).

6. Q. Bao, H. Zhang, B. Wang, Z. Ni, C. Lim, Y. Wang, D. Y. Tang, and K. P. Loh, Nat. Photonics 5, 411 (2011).

7. J. T. Kim and C. G. Choi, Opt. Express 20, 3556 (2012).

8. K. S. Novoselov, V. I. Fal'ko, L. Colombo, P. R. Gellert, M. G. Schwab, and K. Kim, Nature 490, 192 (2012).

9. R. Alaee, C. Menzel, C. Rockstuhl, and F. Lederer, Opt. Express 20, 18370 (2012).

10. S. A. Mikhailov and K. Ziegler, J. Phys. Condens. Matter 20, 384204 (2008).

11. K. L. Ishikawa, Phys. Rev. B 82, 201402 (2010).

12. A. R. Wright, X. G. Xu, J. C. Cao, and C. Zhang, Appl. Phys. Lett. 95, 072101 (2009).

13. E. Hendry, P. J. Hale, J. Moger, and A. K. Savchenko, Phys. Rev. Lett. 105, 097401 (2010).

14. H. Yang, X. Feng, Q. Wang, H. Huang, W. Chen, A. T. S. Wee, and W. Ji, Nano Lett. 11, 2622 (2011).

15. H. Zhang, S. Virally, Q. Bao, L. K. Ping, S. Massar, N. Godbout, and P. Kockaert, Opt. Lett. 37, 1856 (2012).

16. C. De Angelis, A. Laureti-Palma, G. F. Nalesso, and C. G. Someda, Opt. Quantum Electron. 29, 217 (1997).

17. T. Stauber, N. M. R. Peres, and A. K. Geim, Phys. Rev. B 78, 085432 (2008).

18. G. W. Hanson, IEEE Trans. Antennas Propag. 56, 747 (2008).

19. COMSOL Multiphysics 4.3, http://www.comsol.com.

20. F. H. L. Koppens, D. E. Chang, and F. J. Garcia de Abajo, Nano Lett. 11, 3370 (2011).

21. J. Christensen, A. Manjavacas, S. Thongrattanasiri, F. H. L. Koppens, and F. J. Garcia de Abajo, ACS Nano 6, 431 (2012).

22. A. Martinez, K. Fuse, and S. Yamashita, Appl. Phys. Lett. 99, 121107 (2011)

23. A. Locatelli, A.-D. Capobianco, M. Midrio, S. Boscolo, and C. De Angelis, Opt. Express 20, 28479 (2012). 